\title{
Avaliação Experimental da Configuração do Threshold de Balanceamento de Réplicas no HDFS Balancer
}

\author{
Rhauani Weber Aita Fazul ${ }^{1}$, Patrícia Pitthan Barcelos ${ }^{2}$ \\ ${ }^{1}$ Laboratório de Sistemas de Computação (LSC) \\ ${ }^{2}$ Pós-Graduação em Ciência da Computação (PGCC) \\ Universidade Federal de Santa Maria (UFSM) \\ Santa Maria - RS - Brasil \\ \{rwfazul, pitthan\}@inf.ufsm.br
}

\begin{abstract}
Resumo. O HDFS Balancer opera através de um threshold que determina o nível de equilíbrio a ser atingindo com a redistribuição dos dados. Definir um threshold ideal, entretanto, apresenta-se com um desafio para o administrador do sistema. Este trabalho analisa o comportamento do HDFS através de experimentos com variações na configuração do threshold. Os resultados demostram as melhorias de desempenho impulsionadas pelo balanceamento do cluster.
\end{abstract}

\section{Introdução}

A replicação de dados é fundamental para o funcionamento do Sistema de Arquivos Distribuído do Apache Hadoop! o HDFS. Para o armazenamento das réplicas nos DataNodes (DNs), o servidor mestre do HDFS (o NameNode) segue uma Política de Posicionamento de Réplicas (PPR), que fornece um bom equilíbrio entre confiabilidade e disponibilidade dos dados [White 2015]. De todo modo, nem sempre é possível impedir o desbalanceamento na distribuição das réplicas no cluster.

O HDFS Balancer [Shvachko et al. 2010] é uma solução disponibilizada pelo Hadoop que redistribui os dados já armazenados no sistema de arquivos visando o balanceamento de réplicas. Além de definir o momento adequado para a execução do HDFS Balancer, o administrador do sistema deve configurar o threshold de balanceamento. $\mathrm{O}$ threshold controla o funcionamento da ferramenta ao determinar quando o cluster pode ou não ser considerado balanceado. Escolher um bom valor para o threshold é essencial para otimizar a operação de balanceamento de réplicas. Thresholds menores permitem atingir um maior equilíbrio, porém demandam um maior esforço para a movimentação dos dados. Já thresholds maiores reduzem o tempo de execução e a largura de banda consumida pelo balanceador, entretanto resultam em uma distribuição menos equilibrada.

Este trabalho analisa a influência do threshold de balanceamento no funcionamento do HDF Balancer e no desempenho do sistema de arquivos do Hadoop. Para tal, diferentes experimentos com variações no valor do threshold foram conduzidos. Com isso, permite-se investigar as possíveis otimizações impulsionadas pelo equilíbrio na distribuição das réplicas e evidenciar o custo demandado para tal operação.

O artigo está organizado em cinco seções. A Seção 2 descreve as principais causas e os problemas do desbalanceamento no HDFS. A Seção 3 apresenta o balanceador de réplicas nativo do Apache Hadoop. A Seção 4 exibe e discute os resultados obtidos. Por fim, a Seção 5 aponta as considerações finais e direciona os trabalhos futuros.

1https://hadoop.apache.org/ 


\section{Desbalanceamento de Réplicas}

O HDFS implementa uma estratégia de armazenamento que consiste na segmentação dos arquivos em blocos de dados de tamanho fixo, que são replicados com base em um Fator de Replicação (FR) e distribuídos através do cluster. Embora haja um esforço em manter um balanceamento mínimo no posicionamento das réplicas entre os DNs, nem sempre é possível impedir o desequilíbrio. Dentre as principais causas do desbalanceamento de réplicas no HDFS estão [Hortonworks 2019]: (i) a adição de novos DNs ao cluster; (ii) o comportamento da aplicação do cliente; e (iii) a alocação dos blocos satisfazendo a PPR.

O Hadoop explora a localidade dos dados ao mover as tarefas de computação para onde as réplicas estão armazenadas, evitando mover os dados em si e possibilitando que o acesso/processamento seja feito localmente [White 2015]. A medida que o desbalanceamento se intensifica - além de gerar sobrecarga para os DNs com maior utilização -, a localidade é afetada e a largura de banda do cluster passa a ser consumida para realizar transferência de dados, prejudicando o desempenho de aplicações focadas em entrada e saída (E/S) intensiva. Para mitigar esses problemas, o Hadoop disponibiliza uma solução voltada ao balanceamento de réplicas no HDFS, conforme apresentado na Seção 3.

\section{HDFS Balancer}

O HDFS Balancer [Shvachko et al. 2010] é uma ferramenta integrada na distribuição do Hadoop responsável pela análise do posicionamento dos blocos presentes no sistema de arquivos, cabendo a ele tomar as decisões referentes à redistribuição de dados entre os DNs. A partir de sua política de execução padrão, o HDFS Balancer opera iterativamente movimentando blocos de DNs que apresentarem uma alta utilização (origem) para DNs que possuírem um menor volume de dados armazenado (destino) [White 2015]. A execução da ferramenta é disparada sob demanda pelo administrador do cluster.

A operação do balanceador é guiada por um threshold (porcentagem no intervalo de $0 \%$ a 100\%), que é passado como parâmetro para sua execução. Um mesmo DN pode possuir um ou mais dispositivos de armazenamento de diferentes tipos (e.g. disco rígido e SSD). Sendo $G_{i, t}$ o grupo de dispositivos do tipo $t$ de um determinado DN, o threshold limita a diferença máxima que a utilização do $G_{i, t}\left(U_{i, t}\right)$ e a utilização média dos dispositivos do tipo $t$ do cluster $\left(U_{\mu, t}\right)$ pode assumir [Hortonworks 2019]. Quando a utilização de todos os grupos estiver dentro dos limites inferior e superior determinados com base no threshold (i.e., $U_{\mu, t}-$ threshold e $U_{\mu, t}+$ threshold), o cluster é tido como balanceado.

Ao reduzir o threshold aumenta-se o nível de equilíbrio na distribuição das réplicas no cluster, todavia maior o esforço demandado, em termos de processamento e de transferência de dados, para efetuar o balanceamento. A seguir, na Seção 4 , investiga-se o impacto do threshold na operação do balanceador e no funcionamento do HDFS.

\section{Experimentos e Discussão}

A experimentação foi realizada na plataforma GRID'5000 ${ }^{2}$ com o Hadoop (versão 2.9.2) operando em modo totalmente distribuído. O ambiente de testes consistiu em 10 nodos

\footnotetext{
${ }^{2}$ Grid'5000 é uma plataforma para experimentos apoiada por um grupo de interesses científicos hospedado pelo Inria e incluindo CNRS, RENATER e diversas Universidades, bem como outras organizações (mais detalhes em https://wWw.grid5000.fr)
} 
(modelo Dell PowerEdge R640) configurados no cluster gros do site Nancy, cada um com 1 processador Intel Xeon Gold 5220 (Cascade Lake-SP, 2.20GHz, 18 cores/CPU), 96GB de memória RAM, capacidade de armazenamento SDD (SATA) de 480GB e 2 conexões Ethernet de 25Gbps cada, executando uma distribuição Debian 10 (buster).

Para a carga dos dados utilizou-se o TestDF S IO [White 2015], um benchmark distribuído que testa o desempenho do HDFS com operações de E/S intensivas. Com o TestDFSIO, foram escritos 25 arquivos de $25 \mathrm{~GB}$ cada e FR padrão de 3 , totalizando um volume de dados de 1,85TB. Após a escrita, o sistema de arquivos ficou com uma utilização média de 50,55\%. De forma a avaliar possíveis melhorias de desempenho impulsionadas pelo equilíbrio de réplicas, avaliou-se o comportamento do HDFS com a distribuição dos dados baseada na PPR (i.e., sem balanceamento) e após a execução do HDF S Balancer considerando cinco cenários com configurações distintas do threshold de balanceamento, sendo eles: (i) $15 \%$; (ii) $12,5 \%$ (iii) $10 \%$; (iv) $7,5 \%$; e (v) $5 \%$.

A Tabela 1 apresenta, para cada um dos DNs, a sua ocupação em GB $\left(O_{G B}\right)$ e a porcentagem de utilização $\left(U_{\%}\right)$ em cada um dos cenários de teste. Para o cenário sem balanceamento, esses valores equivalem ao estado do HDFS após a escrita dos arquivos e a distribuição dos blocos baseada na PPR. Para os demais cenários, os valores representam o estado de cada um dos DNs do cluster após a execução do HDFS Balancer com a respectiva configuração de threshold $(T h)$. Observa-se como, em cada um dos cenários com balanceamento de réplicas, a utilização final dos DNs passa a respeitar os limites inferior $\left(U_{\mu, S S D}-\right.$ threshold, i.e., $50,55 \%$ - Th) e superior $\left(U_{\mu, S S D}+\right.$ threshold, i.e., $50,55 \%+T h)$ considerados pelo balanceador.

Tabela 1. Ocupação $\left(O_{G B}\right)$ e utilização $\left(U_{\%}\right)$ dos nodos em cada cenário de teste.

\begin{tabular}{|c|c|c|c|c|c|c|c|c|c|c|c|c|}
\hline \multirow{2}{*}{$\mathbf{D N}$} & \multicolumn{2}{|c|}{ sem bal. } & \multicolumn{2}{|c|}{$T h=15 \%$} & \multicolumn{2}{|c|}{$T h=12,5 \%$} & \multicolumn{2}{|c|}{$T h=10 \%$} & \multicolumn{2}{|c|}{$T h=7,5 \%$} & \multicolumn{2}{|c|}{$T h=5 \%$} \\
\hline & $O_{G B}$ & $U_{\%}$ & $O_{G B}$ & $U_{\%}$ & $O_{G B}$ & $U_{\%}$ & $O_{G B}$ & $U_{\%}$ & $O_{G B}$ & $U_{\%}$ & $O_{G B}$ & $U_{\%}$ \\
\hline $\mathrm{N}_{01}$ &  & 5.17 & & 0 & 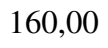 & 0 & 161,62 &  & & 45,14 & 2 & 45,5 \\
\hline $\mathrm{DN}_{02}$ & 142,34 & 36,03 & 1 & 38,52 & & 50,00 & & 41,26 & & 47,99 & 78 & 49,56 \\
\hline $\mathrm{DN}_{03}$ & 144,19 & 36,50 & 162 & 41,07 & 237,60 & 60,14 & 18 & 47,00 & 17 & 43,29 & 19. & 49,56 \\
\hline $\mathrm{DN}_{04}$ & 191,16 & 48,39 & 191 & 48,49 & 164 & 41,65 & 213 & 53,95 & & 52,09 & 30 & 46,14 \\
\hline $\mathrm{DN}_{05}$ & 140,33 & 35,52 & 244 & 61,90 & 235 & 59,57 & 173 & 43,99 & 200 & 50,79 & 180,25 & 45,62 \\
\hline $\mathrm{DN}_{06}$ & & 36,86 & & 60,75 & & 39,09 & & 56,73 & & 54,11 & & 50,64 \\
\hline $\mathrm{DN}_{07}$ & 259,40 & 65,66 & 142,74 & 36,13 & 185,61 & 46,98 & 222,48 & 56,31 & 173,56 & 43,93 & 203,48 & 51,50 \\
\hline $\mathrm{DN}_{08}$ & 286,38 & 72,49 & 220,34 & 55,77 & 166,67 & 42,19 & 160,38 & 40,60 & 213,16 & 53,95 & 184,82 & 46,78 \\
\hline $\mathrm{DN}_{09}$ & 293,07 & 74,18 & 149,94 & 37,95 & 235,46 & 59,60 & 219,33 & 55,52 & 172,42 & 43,64 & 190,34 & 48,18 \\
\hline $\mathrm{DN}_{10}$ & 150,63 & 38,13 & 246,42 & 62,37 & 158,11 & 40,02 & 169,32 & 42,86 & 185,12 & 46,86 & 180,16 & 45,60 \\
\hline
\end{tabular}

Para a análise de desempenho no HDFS, realizou-se, em cada cenário de teste, 15 execuções distintas do benchmark TestDFSIO destinadas à leitura total dos dados armazenados no sistema. A Tabela 2 exibe as médias aritméticas dos valores de tempo de execução, throughput de leitura e taxa de E/S alcançados nas 15 execuções. Adicionalmente, para cada uma dessas três métricas, exibe-se a variação percentual dos cenários com balanceamento em relação ao cenário sem balanceamento. A variação percentual é dada pela equação $\left(\left(T_{b}-T_{a}\right) / T_{a} \times 100\right)$, onde $T_{a}$ e $T_{b}$ equivalem, respectivamente, às médias da métrica em análise no cenário sem balanceamento e com o uso do balanceador com o respectivo threshold $(T h)$. Quando negativa, a variação obtida representa uma redução. Dessa forma, percebe-se uma relação entre o desempenho do HDFS e o 
equilíbrio das réplicas armazenadas no sistema (quanto menor o threshold, maior o nível de balanceamento atingido). Assim, com a localidade dos dados podendo ser melhor explorada, é possível aprimorar o funcionamento do HDFS ao reduzir o tempo necessário para a leitura dos dados e aumentar o throughput e taxa média de E/S do sistema.

Tabela 2. Comportamento do HDFS em cada cenário de teste.

\begin{tabular}{c|c|c|c|c|c|c}
\hline Métrica & sem bal. & Th = 15\% & Th= 12,5\% & Th= 10\% & Th = 7,5\% & Th = 5\% \\
\hline Tempo de execução (s) & 258,85 & 233,51 & 223,51 & 212,69 & 207,94 & 192,09 \\
Variação percentual (\%) & - & $-9,79$ & $-13,65$ & $-17,83$ & $-19,67$ & $-25,79$ \\
\hline Throughput médio (MB/s) & 152,62 & 164,42 & 169,74 & 177,14 & 182,04 & 190,35 \\
Variação percentual (\%) & - & 7,73 & 11,22 & 16,07 & 19,28 & 24,72 \\
\hline Taxa média de E/S (MB/s) & 161,79 & 172,80 & 178,56 & 186,41 & 189,95 & 201,24 \\
Variação percentual (\%) & - & 6,81 & 10,37 & 15,22 & 17,41 & 24,38 \\
\hline Tempo de balanceamento (s) & - & 4015,76 & 4933,29 & 5967,74 & 6234,57 & 9867,83 \\
Dados movimentados (GB) & - & 52,75 & 96,38 & 114,38 & 142,00 & 211,88 \\
\hline
\end{tabular}

Ao final da Tabela 2 exibe-se o tempo de execução do HDFS Balancer (em segundos) e o volume de dados transferidos entre os DNs durante a operação de balanceamento. Quanto menor o threshold maior o esforço necessário (em tempo de execução e largura de banda consumida) para equilibrar a distribuição das réplicas no cluster. Sendo assim, cabe ao administrador do sistema avaliar o trade-off entre as melhorias desempenho impulsionadas pelo balanceamento de réplicas no HDFS e custo para sua operação.

\section{Considerações Finais}

Este trabalho analisou a influência do balanceamento de réplicas no funcionamento do HDFS. Os resultados obtidos demostraram que a execução do HDFS Balancer com valores de threshold menores permite maiores otimizações no desempenho do sistema, porém demandando maior tempo para o balanceamento e realizando um maior número de transferências de dados entre os nodos do cluster. Espera-se que, ao evidenciar o tradeoff entre o desempenho impulsionado e o custo da operação do HDFS Balancer nos experimentos idealizados, seja possível auxiliar a tomada de decisão de administradores de clusters HDFS para a configuração ideal do threhsold de balanceamento.

Trabalhos futuros envolvem novos experimentos com variações na configuração do balanceador nativo do HDFS, tais como a largura de banda máxima destinada ao balanceamento em cada nodo, o número máximo de transferências concorrentes e a quantidade de threads para realizar a redistribuição das réplicas. Adicionalmente, pretende-se avaliar a aplicabilidade do HDFS Balancer com as configurações recomendadas apresentadas em [Hortonworks 2019] para os modos de execução background e fast.

\section{Referências}

Hortonworks (2019). "Balancing data across an HDFS cluster". https://docs. cloudera.com/HDPDocuments/HDP3/HDP-3.1.4/data-storage/ content/balancing_data_across_hdfs_cluster.html. Dezembro.

Shvachko, K., Kuang, H., Radia, S., and Chansler, R. (2010). The hadoop distributed file system. In Symposium on Mass Storage Systems and Technologies, pages 1-10. IEEE.

White, T. (2015). Hadoop: The Definitive Guide. O’Reilly Media, Inc., 4 edition. 